\title{
Material optimisation in dual particle detectors by comparing advanced scintillating materials using two Monte Carlo codes
}

\author{
H. Al Hamrashdi ${ }^{1}$, D. Cheneler ${ }^{1}$ and S. D. Monk ${ }^{1}$ \\ ${ }^{1}$ Department of Engineering, Lancaster University, Lancaster, United Kingdom \\ LA1 4YW
}

\begin{abstract}
A new generation of scintillating materials have been recently developed in the radiation-imaging field offering very promising dual particle detection abilities. Here, four different scintillating materials (Cs2LiYCl6:Ce (CLYC), 95\% ${ }^{6} \mathrm{Li}$ enriched $\mathrm{Cs}_{2} \mathrm{LiYCl}_{6}$ :Ce (CLYC-6), natural Li-glass scintillator (GS10) and liquid scintillator EJ-309) have been characterized for their abilities to attenuate thermal neutrons, fast neutrons and gamma-rays. Recent studies regarding these materials overlook these fundamental characteristics, which can directly affect the design process of advanced imaging systems such as Compton cameras and dual particle imaging systems. The response of each featured material to these three types of radiation fields was simulated with two different Monte Carlo codes, MCNP6 and Geant4. The results indicated that among these four materials, natural Li-glass scintillator (GS10) has the highest thermal neutron detection efficiency and the highest elastic scattering efficiencies. However, the attenuation of fast neutrons was found to be the most severe in EJ-309 liquid scintillator. When gamma-rays are considered, it was found that the mass attenuation coefficient of CLYC and CLYC-6 is the highest of the four materials considered when energies lower than $1 \mathrm{MeV}$ are incident. It is intended that this work will lead to the design and the build of an advanced prototype three stage Compton Camera which will be sensitive to both neutrons and Gamma rays.
\end{abstract}

Key words: Neutron imaging; gamma-ray imaging; MCNP6; Geant4; Cs2LiYCl6:Ce (CLYC), 95\% ${ }^{6}$ Li enriched Cs2LiYCl6:Ce (CLYC-6), natural Li-glass (GS10) and EJ-309 liquid scintillator.

Areas of Novelty: This work compares and contrasts how four different scintillators from four different families interact with thermal neutrons, fast neutrons and gamma-ray using two different simulations toolkits, MCNP6 and Geant4. The work characterizes the materials in terms of their ability to interact favorably with neutrons and gamma-rays.

\section{Introduction}

Accurate localization and characterization of radiation sources is essential in many fields including border security, nuclear security, counter-terrorism, medical imaging as well as within nuclear site decommissioning. There are a number of materials and detectors used in localizing and characterizing radiation sources that emit single mode radiation fields [1-3]. However, the real challenge in radiation detection research field is the development of an optimal detecting material that is capable of detecting both highly penetrating neutrons and gamma-rays.

In the last two decades, the search for high performance scintillators has led to the discovery of new scintillating materials [3,4], some of which are sensitive to both neutrons and gamma-rays. Examples are Elpasolite scintillators [5-10], lithium based glass scintillators [11-16], some classes of liquid scintillators [11, 17-19] and plastic scintillators [20, 21]. An interesting example from the Elpasolite family is the $\mathrm{Cs}_{2} \mathrm{LiYCl}_{6}$ : $\mathrm{Ce}$ (CLYC) scintillator, considered to be one of the most promising inorganic scintillators with an excellent energy resolution of less than $5 \%$ at $662 \mathrm{keV}[4,22]$. The light yield photons of CLYC (with a Ce dopant concentration of $0.1 \%$ ) is estimated to be 20,000 photons/MeV for gamma-rays and 70,000 photons/n for thermal neutrons. In addition, the crystal is sensitive to both thermal and fast neutrons $[4,23,24]$. Enriching the crystal with the ${ }^{6} \mathrm{Li}$ isotope can tone the sensitivity of the detector towards thermal neutrons. $95 \%{ }^{6} \mathrm{Li}$ enriched CLYC is commonly known as CLYC-6 [6, 25]. Within CLYC, thermal neutron detection is mainly due to ${ }^{6} \mathrm{Li}(\mathrm{n}, \mathrm{t}) \alpha$ interactions (thermal neutron $\sigma_{\text {capture }} \sim 40$ barns, $Q \sim 4.8 \mathrm{MeV}$, negligible gamma emission). Fast neutrons mainly interact through elastic and inelastic scattering although capture reactions are possible as well via the ${ }^{35} \mathrm{Cl}(\mathrm{n}, \mathrm{p}){ }^{35} \mathrm{~S}$ and ${ }^{35} \mathrm{Cl}(\mathrm{n}, \alpha){ }^{35} \mathrm{P}$ reactions. Interaction of gamma-rays in CLYC crystals results in a unique Core to Valence 
Luminescence (CVL) with a small decay time ( 1 ns). This unique CVL is used to distinguish gammarays from neutrons [4].

Lithium based glass ( ${ }^{6} \mathrm{Li}$-glass) scintillators are another interesting example of dual particle scintillators. The light yield is estimated to be $\sim 6,000$ photons $/ \mathrm{n}$ for neutrons and $\sim 4,000$ photons/MeV for gammarays [3], with a decay time estimated to be around $75 \mathrm{~ns}$ for a Ce doped glass scintillator. Enriching the scintillating material with $\mathrm{Li}-6$ can tune up the sensitivity towards thermal neutrons. Examples of Liglass scintillators are Saint-Gobain developed Li-glass scintillators with $6.6 \%$ total lithium content. In this work, natural Li-glass scintillator, known as GS10, will be used in the simulations. This glass scintillator also contains varying amounts of $\mathrm{Ce}_{2} \mathrm{O}_{3}, \mathrm{SiO}_{2}, \mathrm{MgO}, \mathrm{Al}_{2} \mathrm{O}_{3}$, and $\mathrm{Li}_{2} \mathrm{O}$ [26]. As in CLYC, thermal neutrons are mainly detected through ${ }^{6} \mathrm{Li}(\mathrm{n}, \mathrm{t}) \alpha$ interactions. However, in lithium glass, fast neutrons are mainly detected through scattering interactions. Pulse Shape Discrimination (PSD) is frequently used with these materials to differentiate between neutrons and gamma-rays [27-29].

Some classes of liquid and plastic scintillators can also be utilized as multi-modal gamma/neutron detectors. The mixture of scintillator and solvent in any detector mainly depends on the application of the imaging system. This family of scintillators is usually found in applications where fast neutrons spectroscopy is required. In general, liquid scintillators have higher tendency to resist radiation and mechanical damages in comparison with plastic scintillators [17, 18]. An example of such a liquid scintillator might be EJ-309 (Eljen Technologies) which offers superior pulse shape discrimination (PSD) compared to some other liquid and plastic scintillators [30] . It also overcomes many drawbacks in other existing liquid scintillators, such as high toxicity [30]. EJ-309 is characterized by a scintillation efficiency of $\sim 12,300$ photons $/ \mathrm{MeV}$ and decay time of $\sim 3.5 \mathrm{~ns}$ [31]. In general, the light yield of EJ-309 depends on the size of the detector $[32,33]$. The detection of fast neutrons in EJ-309 is mainly due to elastic scattering events with hydrogen and carbon nuclei. In general, liquid scintillators, including EJ309 , can efficiently detect neutrons and gamma-ray. However, at low $(<0.1 \mathrm{MeV})$ energies, the abilities of this class of scintillators to discriminate neutrons and gamma-ray fall dramatically [11].

The ultimate aim of this research is the design of a dual particle imaging system where the characterising and quantifying detection abilities of some selected scintillation materials function of energy and distance are essential. Most of the current studies on dual particle imaging discuss properties closely related to the application of interest [2-4, 23, 34, 35]. A. Giaz, et. al. [4] compares the performance of CLYC-6 and CLYC-7 (99\% ${ }^{7} \mathrm{Li}$ enriched CLYC) using the Time of Flight (TOF) and energy spectrum of fast neutrons. Similarly, C. W. E. van Eijk [34] compares the light yield, density and Ce concentration in different inorganic scintillators. In addition, he compares the scintillation properties of some neutron sensitive scintillators. Other studies discuss the detection properties of the integrated imaging system as one unit. For example, Alexis Poitrasson-Rivière et. al. [35] find the angular resolution experimentally and using MCNP of liquid scintillators based dual particle imaging system. Ayaz-Maierhafer et. al [36] study the angular resolution of a combined neutron gamma imaging system. To this end, this work presents a detailed study on the detection efficiency and interaction probabilities of thermal neutrons, fast neutrons and gamma-ray in four different scintillators: CLYC, CLYC-6, natural Li-glass (GS10) and EJ-309 liquid scintillator. This is undertaken using two Monte Carlo codes, MCNP6.1.0 and Geant4.10.2. These four scintillators where selected because they inherit the best scintillating characteristics of their class of materials while acquiring the most efficient detection abilities. In addition, the study aims to aid the design process of advanced neutron and gamma imaging systems, such as Compton cameras and elastic scattering cameras, using any one of these four scintillators. In section 4, a brief description of the detectors arrangement in the design is provided using the design parameters determined within this work.

\section{Materials and methods}

\subsection{MCNP6.1.0 simulations}

MCNP6.1.0 is a general purpose Monte Carlo radiation-transport code used to track radiation particles over a wide range of energies. The code finds applications in radiation shielding, radiation protection, medical physics, nuclear criticality safety and many others related fields. The code was created by merging two already established codes, MCNP5 and MCNPX, adding new features and capabilities. The models used in MCNP6.1.0 apply all possible nuclear interaction process in simulating real life experiments. In general, the MCNP code uses continuous-energy nuclear and atomic data libraries. For neutrons, the Evaluated Nuclear Data Files (ENDF) system is primarily used along with some other nuclear data libraries. In this work, the most updated version of ENDF (ENDF/B-VII.0) was primarily 
used. The neutrons data table energy range in MCNP6.1.0 starts from $10^{-11} \mathrm{MeV}$ to $20 \mathrm{MeV}$ for all isotopes and up to $150 \mathrm{MeV}$ for some of them [37]. The Evaluated Photon Data Library (EPDL) and the Activation Library (ACTL) compilations from Livermore data libraries are mainly used in the simulation of gamma photon transport. In this work, EPDL is the primary source of data and interaction cross section. Data tables energy range for gamma-ray starts from $1 \mathrm{eV}$ to a few $\mathrm{GeV}$ [37].

\subsection{Geant4 simulations}

Geant4.10.2 is a CERN created Monte Carlo based toolkit used to simulate the transition of particles through matter [38], and finds applications wherever particle interaction in matter is considered important. It covers a wide range of parameters including physics models, geometry and particle tracking. Its comprehensive physics models allow it to be used in complex applications in the fields of nuclear physics, particle physics, medical physics and radiation shielding design. This $\mathrm{C}++$ based open-source software undergoes continuous development by its international developer team [39, 40]. The most recent development in 2016 added a wide range of comprehensive improvements to Geant 4 toolkit. The main features of this new version of Geant 4 is the improved particles tracking and scoring capabilities along with some improvements in geometry models which allow more powerful simulations of real experimental setups. Neutron modeling in the Geant4 toolkit is done through the hadronic model. The main neutrons process listed in the hadronic model are high precision elastic, high precision inelastic, high precision capture and high precision fission. The original model is based on nine data libraries including ENDF/B-VI, JENDL-3 and JEF2.2 [40]. New versions of Geant4 have been utilizing more recent data libraries such as ENDF/B-VII.0 and JENDL-4.0 [40].The energy range for neutron simulation is limited to the available data on these libraries (up to $20 \mathrm{MeV}$ for all isotopes and up to $150 \mathrm{MeV}$ for some isotopes). Gamma modeling in Geant4 is done through Electromagnetic (EM) physics modeling which includes HEP (High Energy Physics) models. The model is based on the Livermore evaluated library [40]. The Geant 4 toolkit covers a wide range of gamma energy from $250 \mathrm{eV}$ up to the TeV range. The last update on the Geant 4 toolkit added new physics and processes to the existing gamma models. Major parts of these modifications add specialized models to two major gamma photon interactions, Pair production and Compton scattering. The modifications were intended improve models accuracy. The results of the new models are compared to NIST values and are reported to be within a tolerance of $10 \%$ $[40]$.

\subsection{Means of comparison and calculations of interest}

Thermal neutron detection is mainly facilitated through capture reactions. Here, the capture efficiency of thermal neutrons in the four scintillators is studied via simulating the passage of thermal neutrons through the scintillating materials as a function of thickness. The definition of absolute efficiency for a particular interaction is the ratio of the number of counts to the number of particles which originated from the source. A sphere of each of the four scintillators with a point source at the center was simulated using both the MCNP6 and GEANT4 codes. In MCNP6.1.0, the F8 tally with special treatment card was used to find the capture efficiency in each component in the four scintillators. The F8 tally provides the user with energy distribution of pulses in the detector region. In Geant4 the simulation was undertaken by counting the number of capture reactions relative to the total number of events.

The study of the response of the materials to fast neutrons was mainly done through investigating elastic scattering interaction probabilities and escaping event probabilities. This was done at four different neutron energies, $1 \mathrm{keV}, 10 \mathrm{keV}, 0.1 \mathrm{MeV}$ and $1 \mathrm{MeV}$. The reaction rate for a flux of neutrons, $\varphi$, incident through a thin layer of matter per unit volume can be described with the following relation [41]

$$
\frac{\text { Reaction Rate }}{\text { volume }}=\Sigma . \varphi
$$

Where $\Sigma$ is the macroscopic cross section $\left(\mathrm{m}^{-1}\right)$. Table 2.1 shows the total cross section and the elastic scattering cross section of CLYC, CLYC-6, natural Li-glass scintillator (GS10) and EJ-309 liquid scintillator based on ENDF/B-VII.0 data library [42]. 
Table 2.1 Total and elastic scattering macroscopic cross sections of CLYC, CLYC-6, natural Li-glass scintillator (GS10) and EJ-309 liquid scintillator.

\begin{tabular}{|c|c|c|c|c|c|c|c|c|}
\hline \multirow[b]{2}{*}{$\begin{array}{c}\text { Energy } \\
(\mathrm{MeV})\end{array}$} & \multicolumn{2}{|c|}{ CLYC } & \multicolumn{2}{|c|}{ CLYC-6 } & \multicolumn{2}{|c|}{ Li-glass (GS10) } & \multicolumn{2}{|c|}{$\begin{array}{l}\text { EJ-309 liquid } \\
\text { scintillator }\end{array}$} \\
\hline & $\begin{array}{l}\text { Total } \\
\left(\mathrm{cm}^{-1}\right)\end{array}$ & $\begin{array}{c}\text { Elastic } \\
\text { scattering } \\
\left(\mathbf{c m}^{-1}\right)\end{array}$ & $\begin{array}{l}\text { Total } \\
\left(\mathrm{cm}^{-1}\right)\end{array}$ & $\begin{array}{c}\text { Elastic } \\
\text { scattering } \\
\left(\mathrm{cm}^{-1}\right)\end{array}$ & $\begin{array}{l}\text { Total } \\
\left(\mathrm{cm}^{-1}\right)\end{array}$ & $\begin{array}{c}\text { Elastic } \\
\text { scattering } \\
\left(\mathbf{c m}^{-1}\right)\end{array}$ & Total $\left(\mathrm{cm}^{-1}\right)$ & $\begin{array}{c}\text { Elastic } \\
\text { scattering } \\
\left(\mathbf{c m}^{-1}\right) \\
\end{array}$ \\
\hline 0.001 & 10.20 & 9.94 & 12.60 & 11.27 & 23.14 & 22.13 & 131.74 & 111.04 \\
\hline 0.01 & 8.81 & 8.08 & 10.20 & 9.85 & 17.22 & 7.11 & 104.91 & 104.91 \\
\hline 0.1 & 7.50 & 7.22 & 8.83 & 8.69 & 15.94 & 8.87 & 69.68 & 69.68 \\
\hline 1 & 13.95 & 12.84 & 15.01 & 14.92 & 27.98 & 6.74 & 23.22 & 23.22 \\
\hline
\end{tabular}

In the work presented here, the response of the material to incident neutrons was studied as a function of thickness. The geometry used in this set of simulations was a cylinder with an infinite radius compared to its length (thickness here) with a point source placed on the face of the cylinder to maximize the entrance dose of fast neutrons into the cylinder. This geometry was chosen to minimize the effect of multiple scattering events. In the MCNP6.1.0 simulations, the PTRAC file option was used in the analysis as in the case of neutrons, the type of interaction taking place in the material needs to be identified; a process which cannot be done using typical tallies. The PTRAC file option allows the user to track the particle of interest through the material. A number of keywords can be added to the PTRAC card that can help identifying the types of interaction along the particles trajectory.

The response of each material to incident gamma-ray was mainly studied through the mass attenuation coefficient. Gamma interaction in matter mainly depends on the energy of the incident photon, the density of the material and the effective atomic number. Therefore, in gamma photon analysis CLYC and CLYC-6 were treated as one material. The effective atomic number according to the power law method is $[43,44]$ :

$$
\mathrm{Z}_{\text {eff }}=\sqrt[2.94]{\sum f_{i} Z_{i}^{2.94}}
$$

Where $f_{i}$ and $Z_{i}$ are the relative electron fraction and the atomic number of the $i^{\text {th }}$ element respectively. The mass attenuation coefficient, $\mu_{\mathrm{m}}\left(\mathrm{cm}^{2} / \mathrm{g}\right)$ measures the probability of interaction in a given material. The analysis of the mass attenuation coefficient is based on attenuation law of gamma-ray [45]:

$$
\mu_{\mathrm{m}}=\mu / \rho=\ln \left(I / I_{o}\right) / x
$$

Where $\mu$ is the linear attenuation coefficient and $\rho$ is the density of the material. $I$ is the attenuated gamma-ray at $x$ and $I_{o}$ is the incident gamma-ray. The mass attenuation coefficient for each material as a function of energy can easily be found within the Geant4 simulation package.

The probability of Compton scattering in each material is calculated as a function of energy as well. For most target materials, the relative probability of Compton scattering occurrence is more dominant in the energy region between $0.1 \mathrm{MeV}$ and $10 \mathrm{MeV}$. The photoelectric effect is more likely to occur at energies lower than $0.1 \mathrm{MeV}$, whereas, Pair Production dominates at energies higher than $10 \mathrm{MeV}$ [45]. Comparing the probability of Compton scattering occurrence in these scintillators is vital in choosing which material is best utilized in Compton scattering based imaging systems.

\section{Results and discussion}

\subsection{Thermal neutrons}

Thermal neutron interaction in the four organic scintillators was modeled using MCNP6.1.0 and Geant4.10.2. In the design process of scintillation based neutron detectors, thermal neutron absorption is the interaction process of major interest, and it is this factor that is compared here over the four organic scintillators. Figure 3.1 shows the thermal neutron intrinsic capture efficiency of as a function of thickness for CLYC, CLYC6, natural Li-glass and an EJ-309 liquid scintillator. The results indicate that intrinsic thermal neutron capturing efficiency increases steadily as a function of thickness before 
saturating for all four materials. Lithium-based scintillators, CLYC, CLYC-6 and Li-glass, require less than $5 \mathrm{~cm}$ of transport through the material in question to reach their maximum intrinsic efficiency.

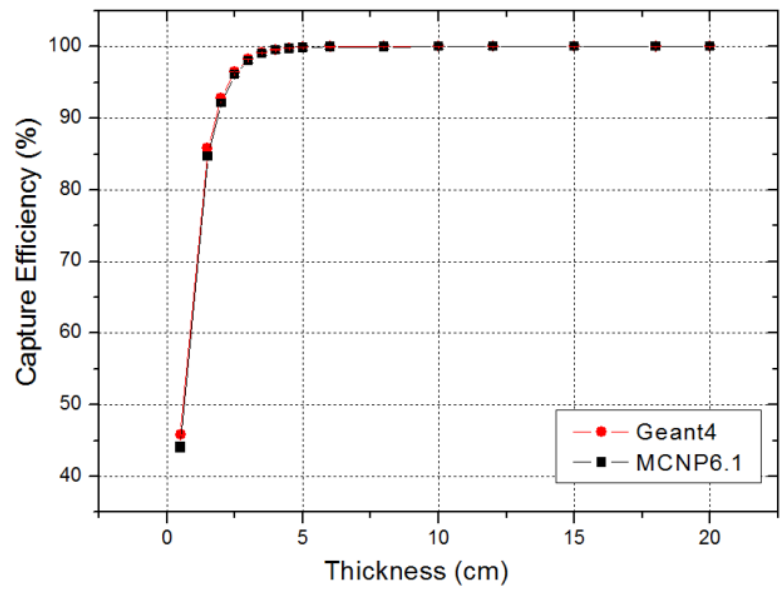

(a)

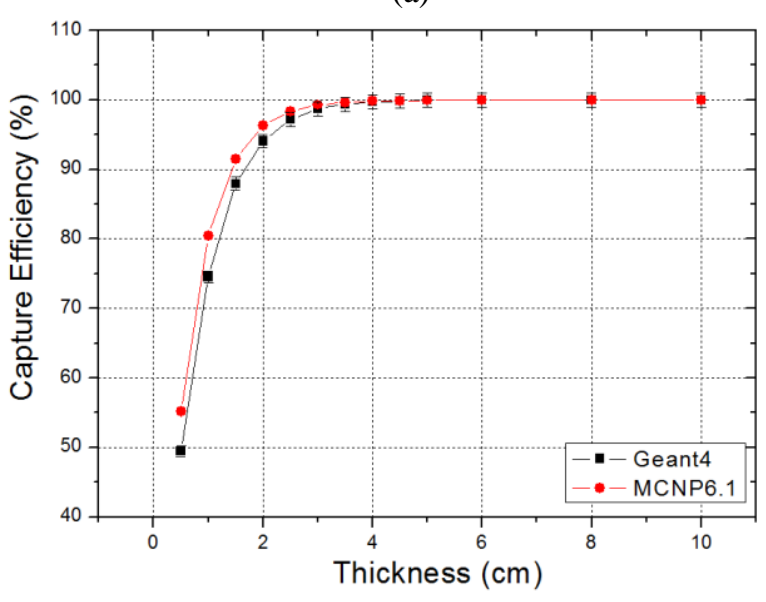

(c)

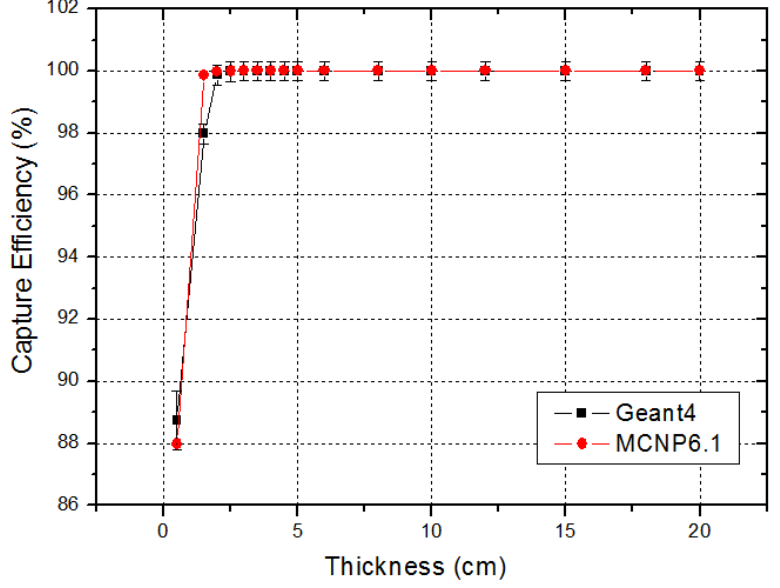

(b)

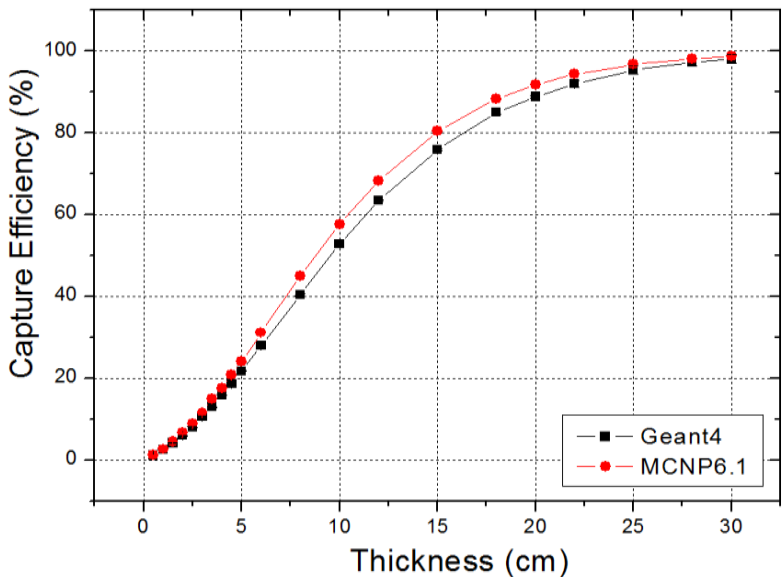

(d)

Figure 3.1. Thermal neutron capturing efficiency as function of thickness of (a) CLYC, (b) CLYC-6, (c) natural Li-glass (GS10) (d) EJ-309 liquid scintillator.

The maximum difference between MCNP6.1.0 and Geant4.10.2 simulations for the four scintillators is less than 5\%. These differences are mainly due to differences in cross sections libraries used by MCNP6.1.0 and Geant4.10.2 [46-50]. Table 3.1 compares thermal neutron capture efficiency of the four scintillators at a thickness of $2 \mathrm{~cm}, 5 \mathrm{~cm}$ and $10 \mathrm{~cm}$ respectively.

Table 3.1 MCNP6.1.0 simulations of thermal neutron capture efficiency in CLYC, CLYC-6, natural Liglass and EJ-309 liquid scintillator.

\begin{tabular}{|c|c|c|c|c|}
\hline $\begin{array}{l}\text { Thickness } \\
\text { (cm) }\end{array}$ & $\begin{array}{l}\text { CLYC } \\
\text { Efficiency } \\
(\%) \text { (capture } \\
\text { in }{ }^{6} \mathbf{L i} \text { ) }\end{array}$ & $\begin{array}{l}\text { CLYC-6 } \\
\text { Efficiency (\%) } \\
\text { (capture in } \\
\left.{ }^{6} \mathbf{L i}\right)\end{array}$ & $\begin{array}{l}\text { Natural } \\
\text { Li-glass efficiency } \\
(\%) \text { (capture in } \\
\left.{ }^{6} \mathrm{Li}\right)\end{array}$ & $\begin{array}{l}\text { Liquid } \\
\text { scintillator } \\
\text { efficiency } \\
(\text { EJ-309) } \\
(\%)\end{array}$ \\
\hline 2 & $\begin{array}{l}92.86 \pm 0.03 \\
(19.89 \pm 0.04)\end{array}$ & $\begin{array}{l}99.98 \pm 0.01 \\
(77.67 \pm 0.04)\end{array}$ & $\begin{array}{l}96.33 \pm 0.01 \\
(96.10 \pm 0.05)\end{array}$ & $5.95 \pm 0.08$ \\
\hline 5 & $\begin{array}{l}99.90 \pm 0.02 \\
(21.40 \pm 0.04)\end{array}$ & $\begin{array}{l}100.01 \pm 0.01 \\
(77.68 \pm 0.04)\end{array}$ & $\begin{array}{l}99.92 \pm 0.01 \\
(96.69 \pm 0.01)\end{array}$ & $21.7 \pm 0.5$ \\
\hline 10 & $\begin{array}{l}100.00 \pm 0.01 \\
(21.42 \pm 0.04)\end{array}$ & $\begin{array}{l}100.00 \pm 0.01 \\
77.68 \pm 0.04)\end{array}$ & $\begin{array}{l}99.95 \pm 0.01 \\
(99.71 \pm 0.01)\end{array}$ & $52.8 \pm 0.2$ \\
\hline
\end{tabular}


Table 3.2 Geant4.10.2 simulations of thermal neutron capture efficiency in CLYC, CLYC-6, natural Liglass and EJ-309 liquid scintillator.

\begin{tabular}{|c|c|c|c|c|}
\hline $\begin{array}{l}\text { Thickness } \\
\text { (cm) }\end{array}$ & $\begin{array}{l}\text { CLYC } \\
\text { Efficiency } \\
(\%) \text { (capture } \\
\left.\text { in }{ }^{6} \mathrm{Li}\right)\end{array}$ & $\begin{array}{l}\text { CLYC-6 } \\
\text { Efficiency } \\
(\%) \text { (capture } \\
\left.\text { in }{ }^{6} \mathrm{Li}\right)\end{array}$ & $\begin{array}{l}\text { Natural } \\
\text { Li-glass } \\
\text { efficiency } \\
(\%) \text { (capture } \\
\left.\text { in }{ }^{6} \mathrm{Li}\right)\end{array}$ & $\begin{array}{l}\text { Liquid } \\
\text { scintillator } \\
\text { efficiency } \\
(\text { EJ-309) } \\
(\%)\end{array}$ \\
\hline 2 & $\begin{array}{l}92.1 \pm 0.3 \\
(23.2 \pm 0.5)\end{array}$ & $\begin{array}{l}99.9 \pm 0.3 \\
(79.7 \pm 0.3)\end{array}$ & $\begin{array}{l}94 \pm 1 \\
(93.9 \pm 0.3)\end{array}$ & $6.0 \pm 0.3$ \\
\hline 5 & $\begin{array}{l}99.8 \pm 0.3 \\
(25.5 \pm 0.5)\end{array}$ & $\begin{array}{l}100.0 \pm 0.3 \\
(79.8 \pm 0.3)\end{array}$ & $\begin{array}{l}100 \pm 1 \\
(99.7 \pm 0.3)\end{array}$ & $21.6 \pm 0.5$ \\
\hline 10 & $\begin{array}{l}100.0 \pm 0.3 \\
(25.5 \pm 0.5)\end{array}$ & $\begin{array}{l}100.0 \pm 0.3 \\
(79.8 \pm 0.3)\end{array}$ & $\begin{array}{l}100 \pm 1 \\
(99.7 \pm 0.3)\end{array}$ & $52.8 \pm 0.7$ \\
\hline
\end{tabular}

Tables 3.1 and 3.2 illustrate that natural CLYC-6 has higher intrinsic capture efficiency of thermal neutrons compared to the other three scintillators followed by CLYC. However, for detection purposes, Li-glass showed higher capture efficiency in ${ }^{6} \mathrm{Li}$. Compared to CLYC and CLYC-6, Li-glass has higher atomic density of ${ }^{6} \mathrm{Li}$ atoms, $1.81 \times 10^{22}$ atoms $/ \mathrm{cm}^{3}$ compared to $1.58 \times 10^{20}$ atoms $/ \mathrm{cm}^{3}$ in CLYC and $1.97 \times 10^{21}$ atoms $/ \mathrm{cm}^{3}$ in CLYC-6.

\subsection{Fast neutrons}

Fast neutron interaction in the four scintillators was simulated at four different energies $(1 \mathrm{keV}, 10 \mathrm{keV}$, $0.1 \mathrm{MeV}$ and $1 \mathrm{MeV}$ ). The results of elastic scattering efficiency, from single interaction events, as well as the escaping percentages as function of thickness are shown from figure 3.2 to figure 3.5. As above, MCNP6.1.0 simulations agree with Geant4.10.2 simulations with discrepancies of less than 5\%. A common trend in all graphs is the exponential decrease of the escaping probability as a function of thickness in all four materials. As energy increases, the probability of escape and elastic scattering slightly vary with thickness. That is mainly because the macroscopic cross section slightly varies over these energies. In general, in CLYC and CLYC-6 the elastic scattering cross section slightly decreases as function of energy. However, at each energy the probability of single elastic scattering slowly increases as function of thickness before starting to decrease again as other types of interaction occur.

Natural Li-glass scintillator exhibits higher attenuation for fast neutrons as compared to CLYC and CLYC-6. This is mainly due to the higher total cross section of Li-glass. The single scattering abilities of fast neutrons is the highest in Li-glass compared to the other three materials for similar reasons. The EJ-309 liquid scintillator exhibits the highest attenuating ability in fast neutrons as a function of thickness across the four specified energies. This is mainly due to the high scattering cross section of the hydrogen atom which causes multiple scattering events which has the effect of slowing down neutrons while they travel through the scintillator. Single scattering probability and escaping probability slightly varies across the four energies. Unlike the CLYC, CLYC-6 and Li-glass based scintillators, the abilities of EJ-309 to cause single elastic scattering decreases exponentially with increasing thickness. This is because other attenuation mechanisms, such as multiple scattering, increase as the thickness of as thickness of the material increases. 


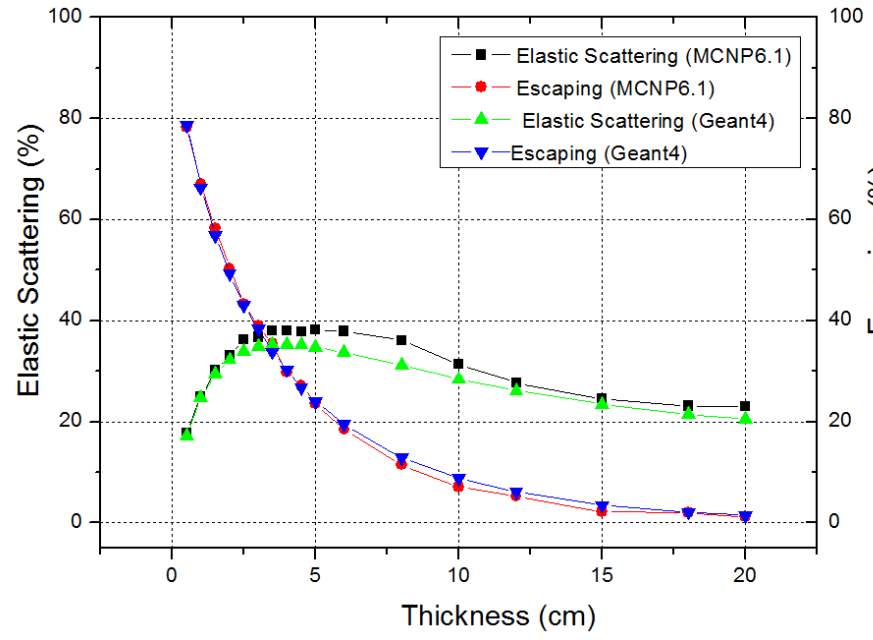

(a)

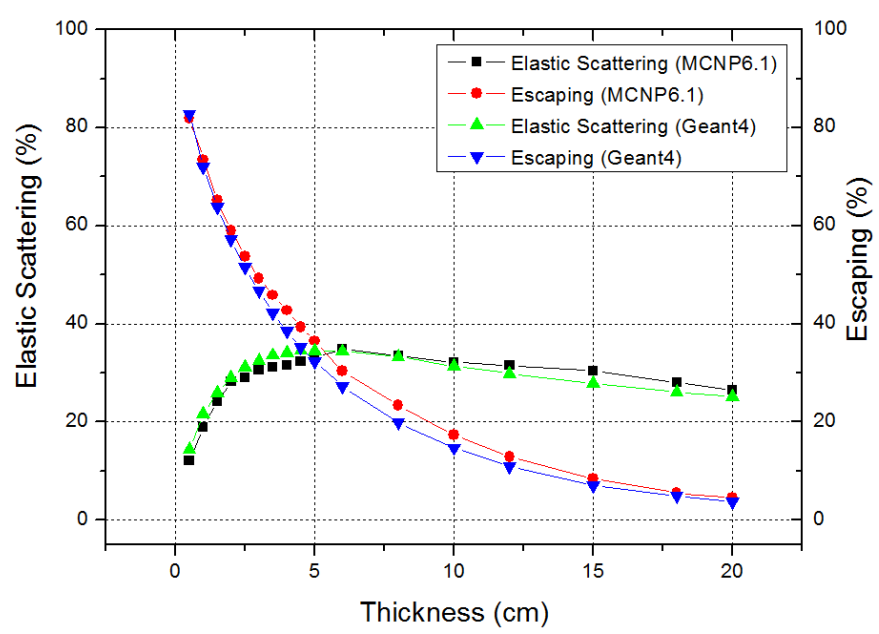

(c)

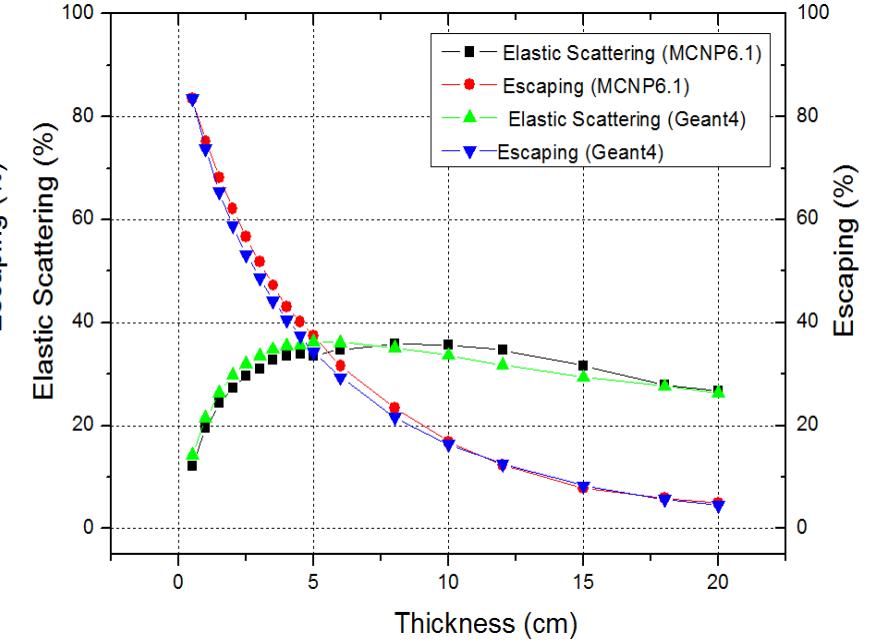

(b)

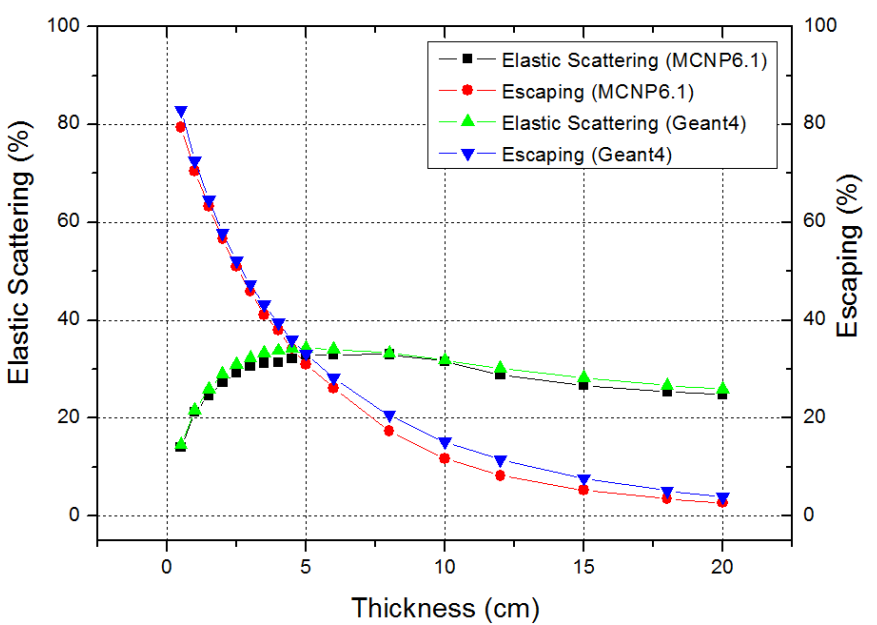

(d)

Figure 3.2. Elastic scattering probability and escaping probability in CLYC at (a) $1 \mathrm{MeV}$, (b) $0.1 \mathrm{MeV}$, (c) $10 \mathrm{keV}$ and (d) $1 \mathrm{keV}$. 


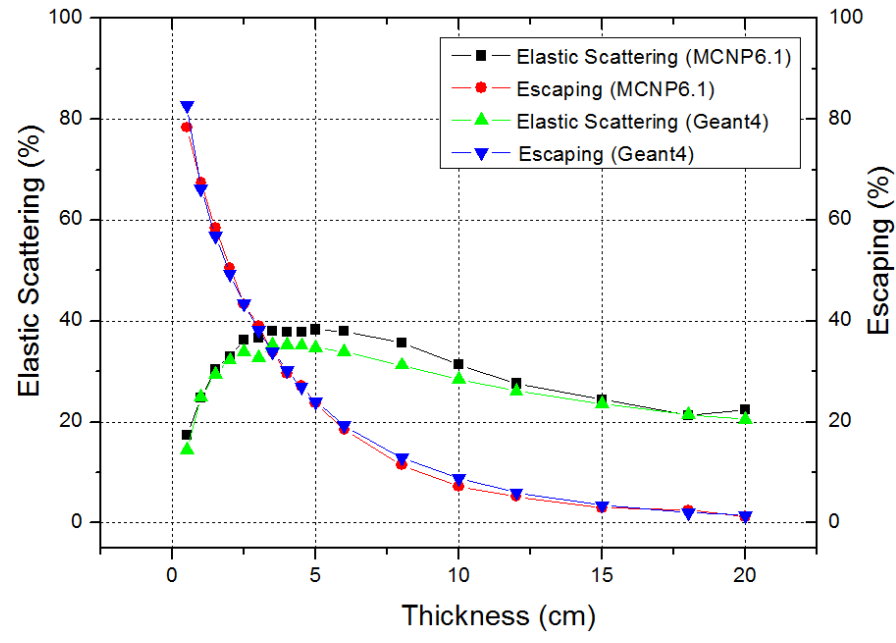

(a)

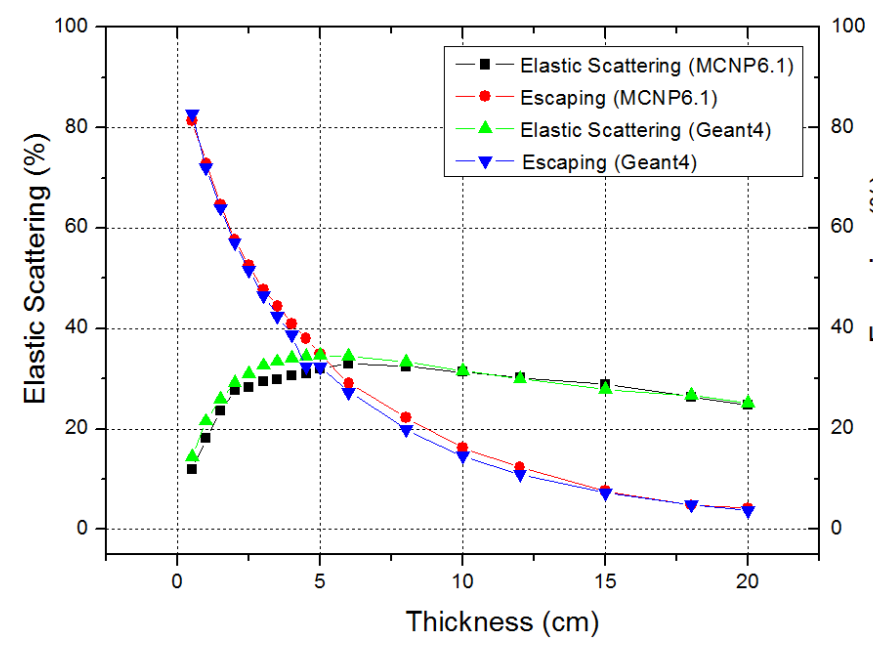

(c)

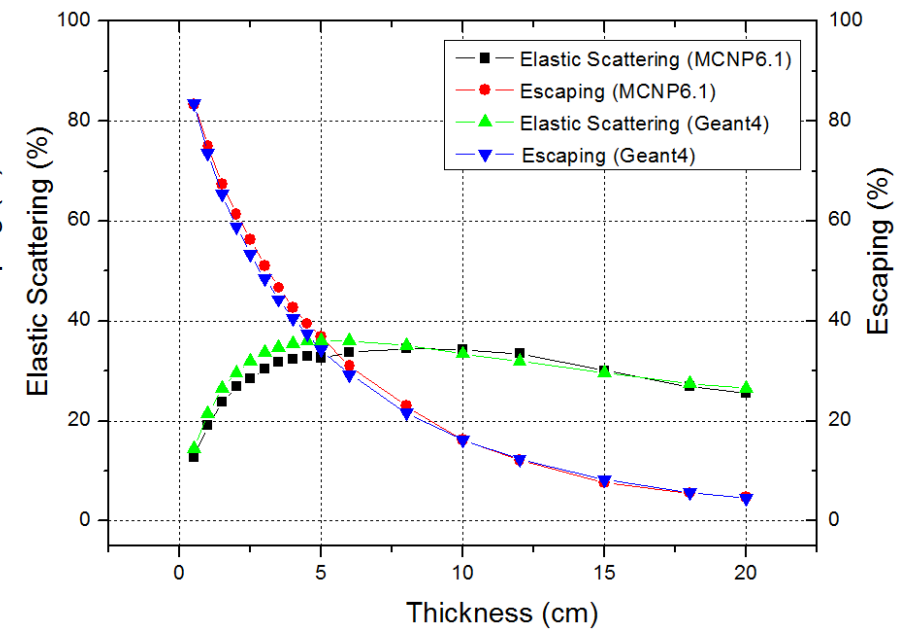

(b)

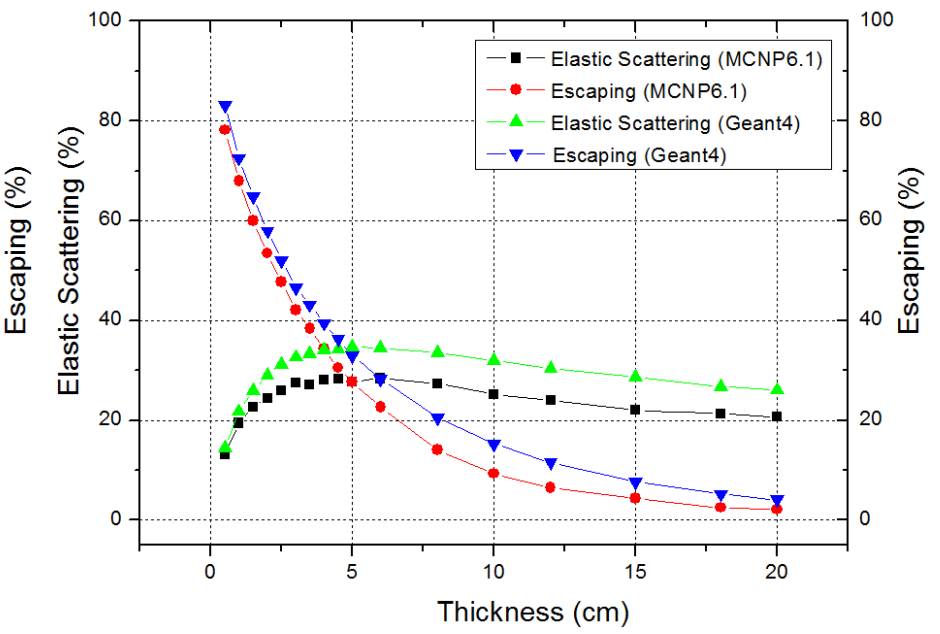

(d)

Figure 3.3. Elastic scattering probability and escaping probability in CLYC-6 at (a) $1 \mathrm{MeV}$, (b) $0.1 \mathrm{MeV}$, (c) $10 \mathrm{keV}$ and (d) $1 \mathrm{keV}$. 


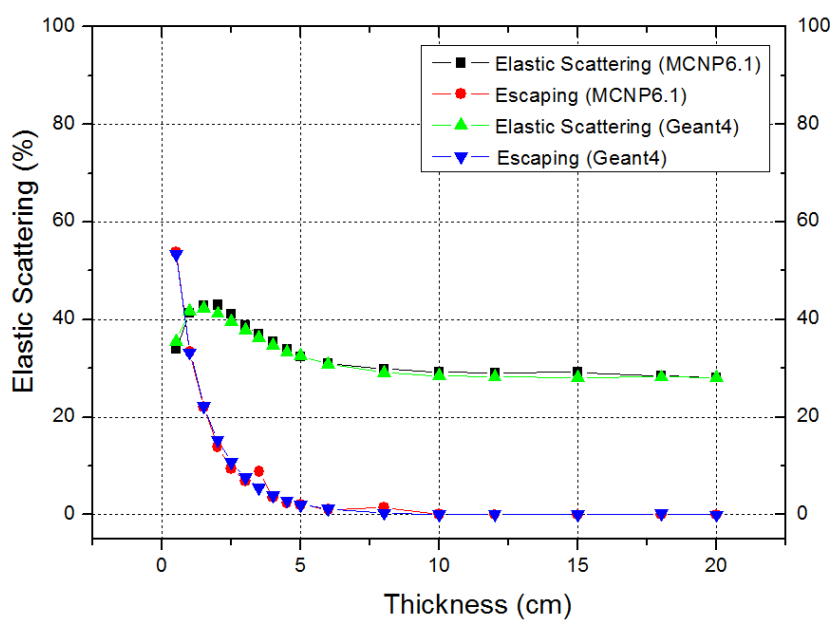

(a)

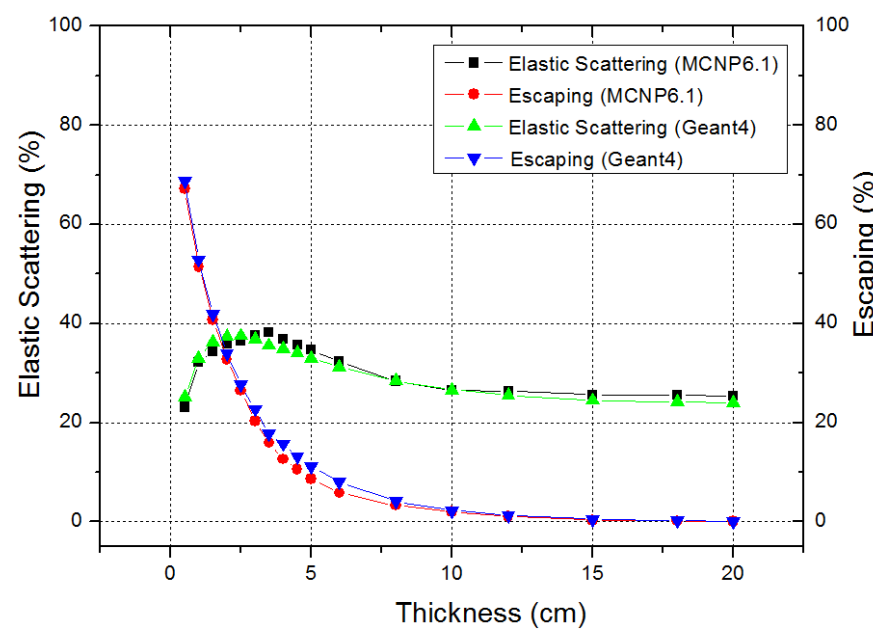

(c)

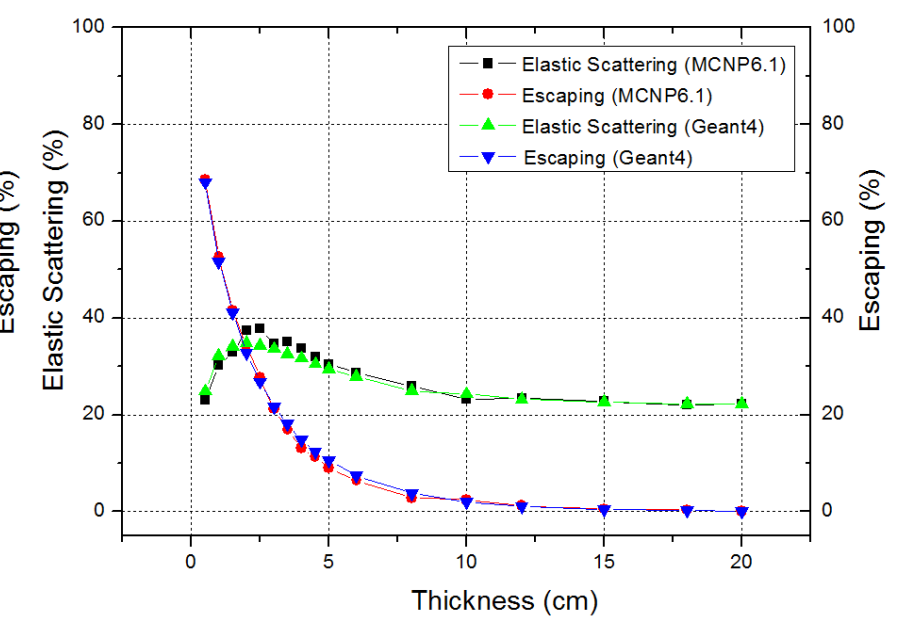

(b)

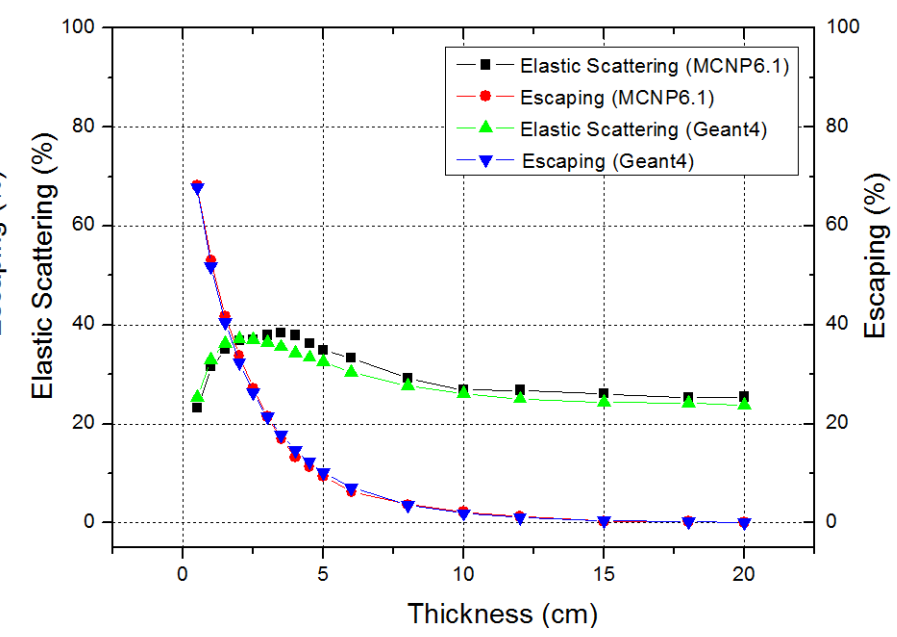

(d)

Figure 3.4. Elastic scattering probability and escaping probability in GS10 natural Li-glass (GS10) at (a) 1 $\mathrm{MeV}$, (b) $0.1 \mathrm{MeV}$, (c) $10 \mathrm{keV}$ and (d) $1 \mathrm{keV}$. 


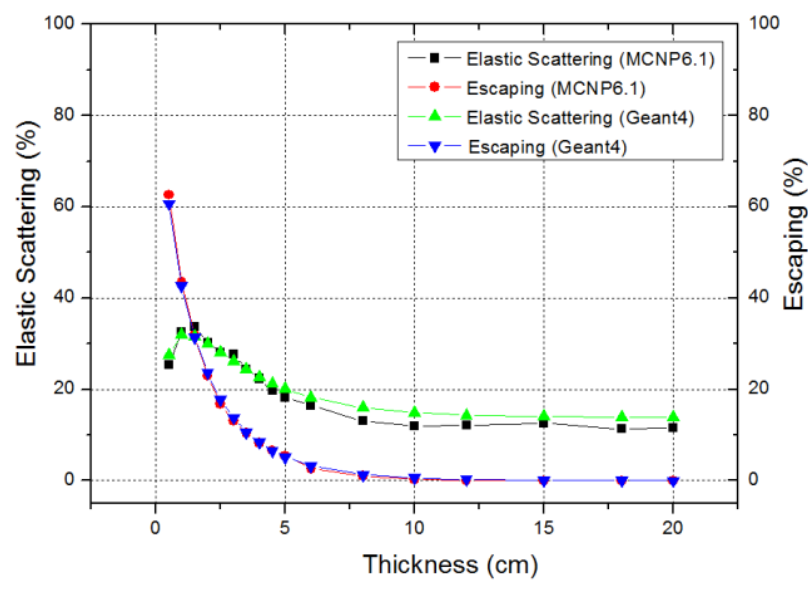

(a)

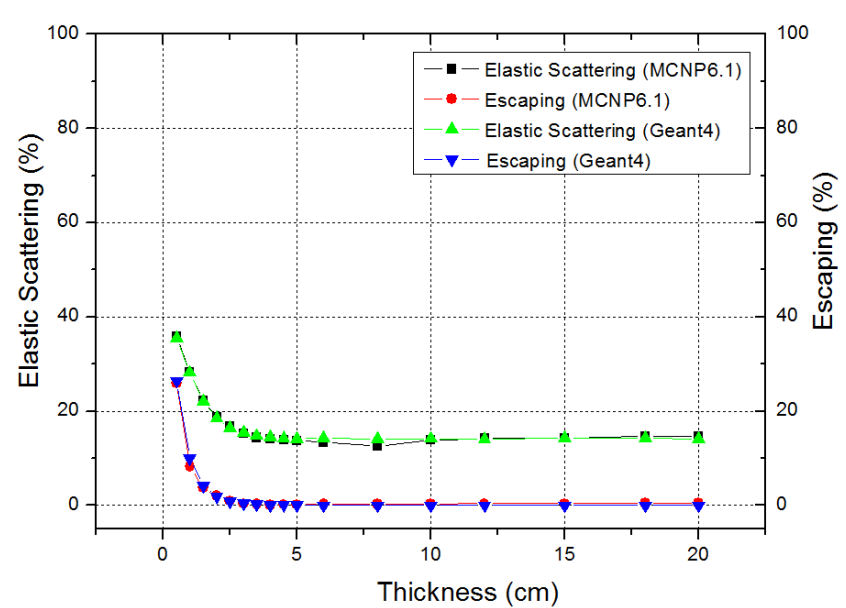

(c)

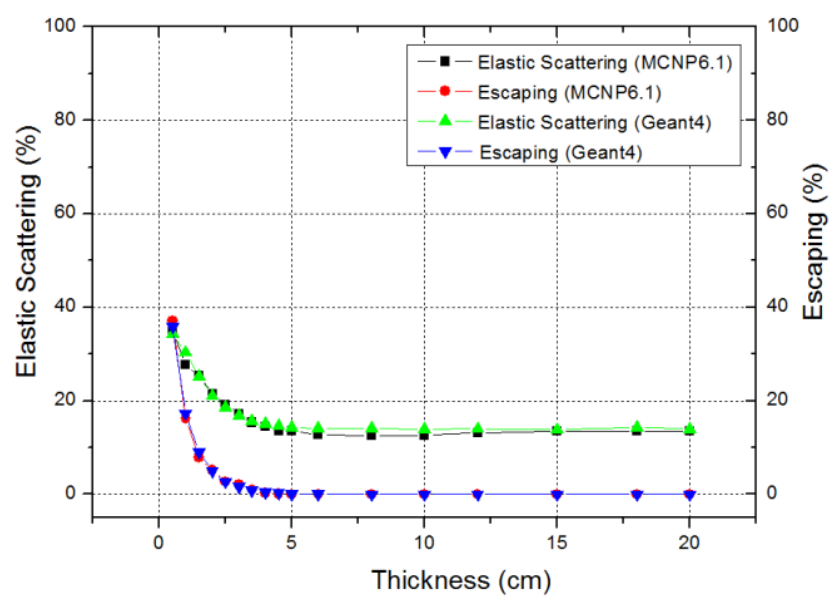

(b)

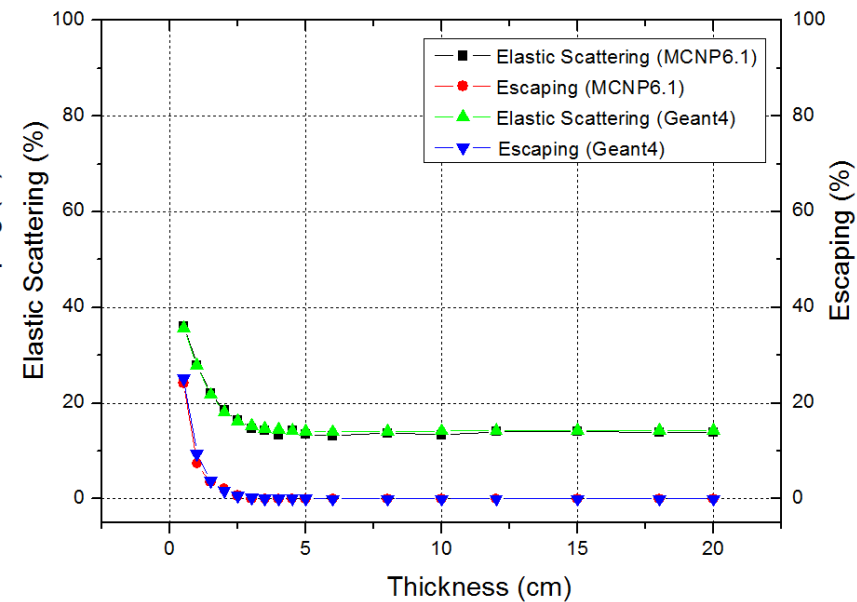

(d)

Figure 3.5. Elastic scattering probability and escaping probability in EJ-309 liquid scintillator at (a) $1 \mathrm{MeV}$, (b) $0.1 \mathrm{MeV}$, (c) $10 \mathrm{keV}$ and (d) $1 \mathrm{keV}$.

Figure 3.6 compares single elastic scattering abilities over $2 \mathrm{~cm}$ considering four different neutron energies; 1 $\mathrm{MeV}, 0.1 \mathrm{MeV}, 10 \mathrm{keV}$ and $1 \mathrm{keV}$. The figure splits elastic scattering events into backscattering efficiency and forward scattering efficiency. The natural Li-glass shows higher elastic scattering abilities in general, with higher backscattering efficiency compared to forward scattering across all four listed energies. CLYC and CLYC-6 show similar elastic scattering abilities with slightly higher forward scattering efficiency compared to backscattering efficiency. Finally, the EJ-309 liquid scintillator exhibits higher backscattering efficiency compared to forward scattering efficiency except at $1 \mathrm{MeV}$. In general, the results of MCNP6.1.0 and Geant4.10.2 simulations agree with attenuation cross sections as reported in table 1.1 . 


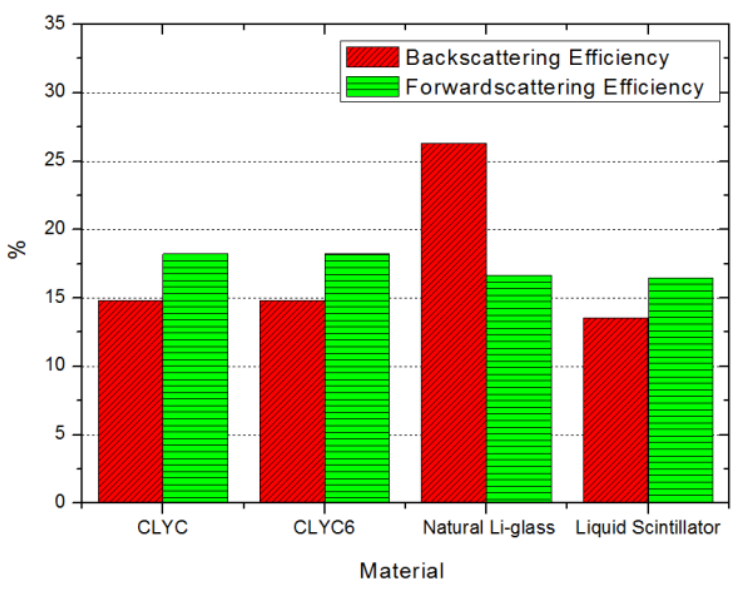

(a)

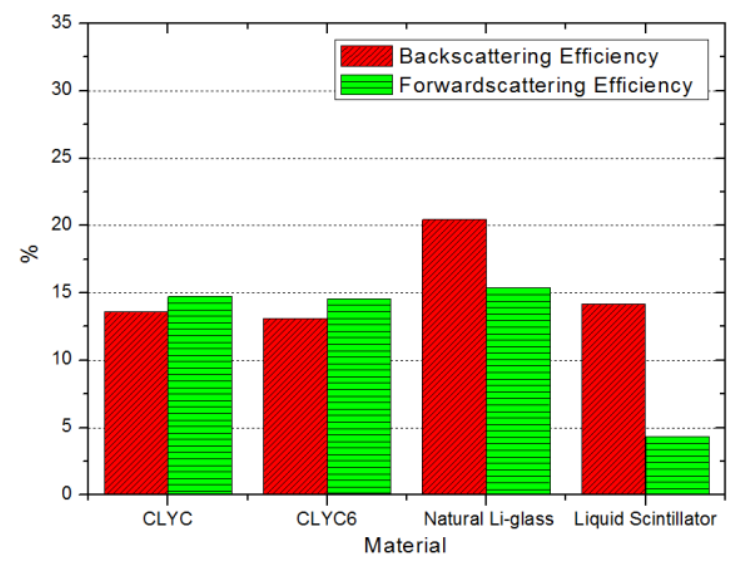

(c)

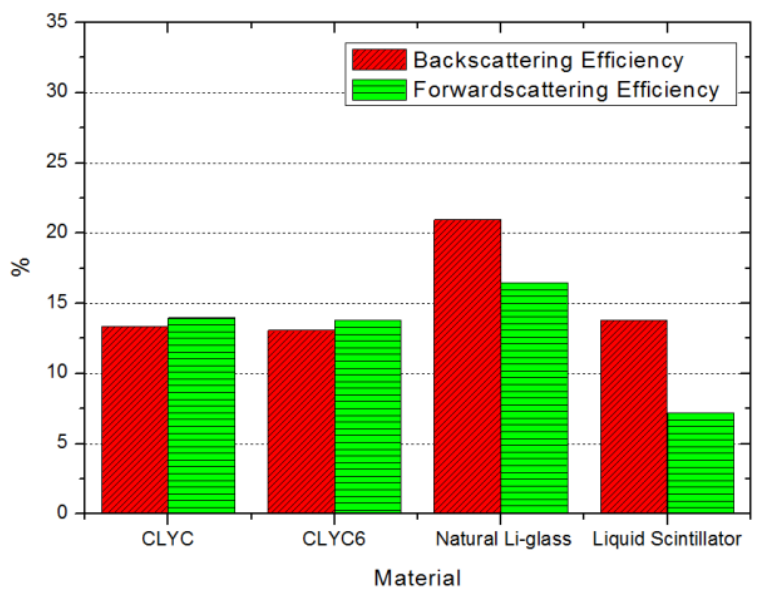

(b)

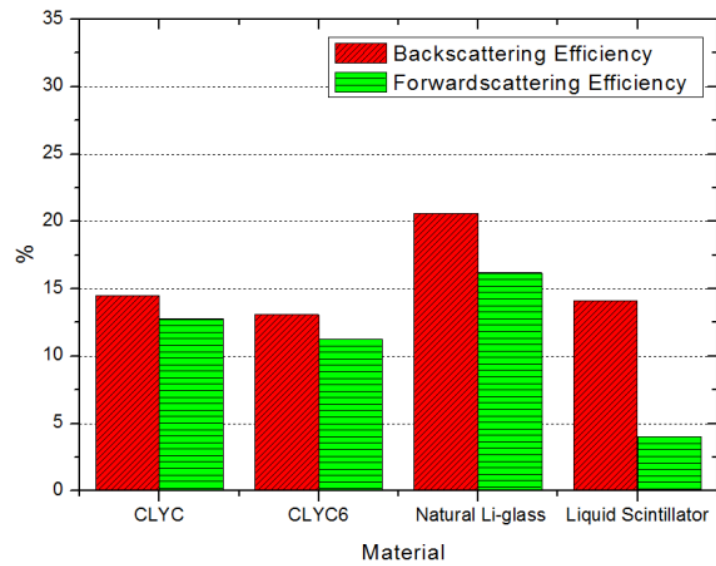

(d)

Figure 3.6. Comparison between backscattering efficiency and forward scattering efficiency of MCNP6.1.0 simulations at (a) $1 \mathrm{MeV}$, (b) $0.1 \mathrm{MeV}$, (C) $10 \mathrm{keV}$ (d) $1 \mathrm{keV}$.

\subsection{Gamma-ray}

Using equation 3 , the mass attenuation coefficient was calculated for each of the four materials. The total mass attenuation coefficients determined by the Geant 4 simulations are shown in figure 3.7. NIST values were extracted using XCOM version 3.1 [51]. Geant4 simulation results are in good agreement with the NIST values. A common feature in all three graphs is the decreasing total mass attenuation coefficient with increasing incident photon energy, which clearly indicated the dependency of the mass attenuation coefficient on energy. This is mainly due to the decrease in the interaction probability between the incident photon and the target material. 


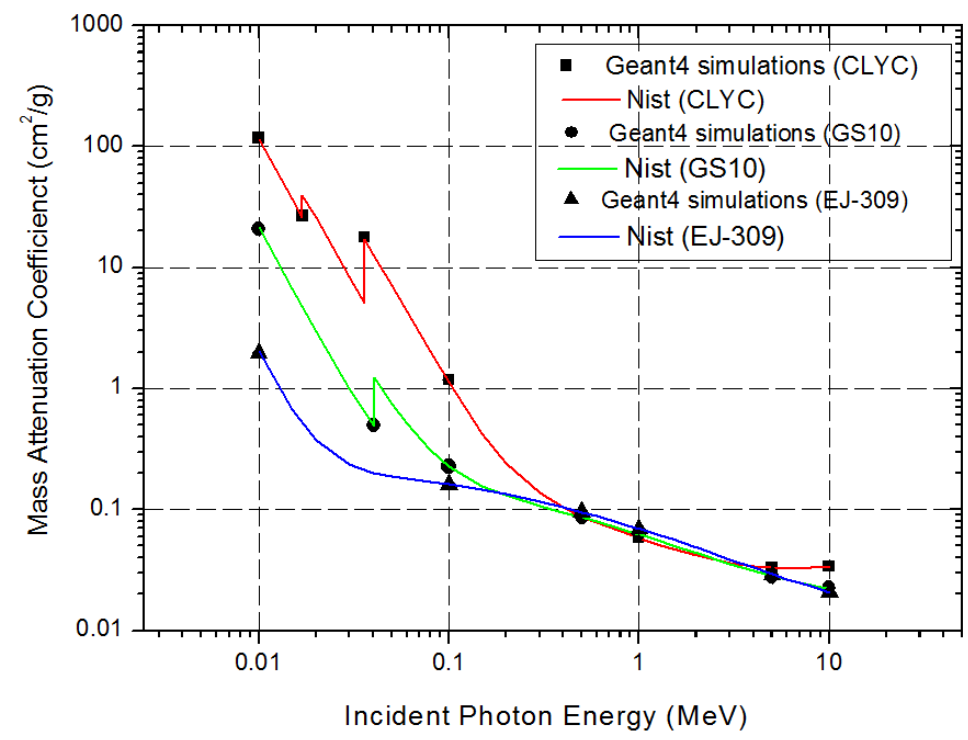

Figure 3.7 Mass attenuation coefficient as function of energy CLYC, natural Li-glass (GS10) and EJ-309 liquid scintillator from Geant 4 simulations and NIST values.

The attenuation abilities of the four listed materials are compared in table 3.3. At energies lower than $0.1 \mathrm{MeV}$ the total mass attenuation coefficient of CLYC and CLYC-6 crystals is higher compared to GS10 and EJ-309. At this energy range, photoelectric absorption is the most common interaction mechanism between gamma-ray and matter. The higher density and the higher effective atomic number of CLYC/CLYC-6 causes a noticeable effect on its attenuation ability compared to the other materials. At higher energies, the total mass attenuation coefficients of the three scintillators decreases as the probability of interaction decreases.

Table 3.3 Comparison of the total mass attenuation coefficient of CLYC/CLYC-6, natural Li-glass (GS10) and liquid scintillator estimated using Geant 4 .

\begin{tabular}{|c|c|c|c|}
\hline $\begin{array}{l}\text { Energy } \\
(\mathrm{MeV})\end{array}$ & $\begin{array}{l}\text { CLYC/CLYC-6 } \\
\left(\mathrm{Z}_{\mathrm{eff}}=42.50\right) \\
\left(\rho=3.31 \mathrm{~g} / \mathrm{cm}^{3}\right)\end{array}$ & $\begin{array}{l}\text { Natural Li-glass } \\
\left(\mathrm{Z}_{\text {eff }}=11.81\right) \\
\left(\rho=2.5 \mathrm{~g} / \mathrm{cm}^{3}\right)\end{array}$ & $\begin{array}{l}\text { Liquid scintillator } \\
\text { (EJ-309) } \\
\left(\mathrm{z}_{\text {eff }}=7.62\right) \\
\left(\rho=0.964 \mathrm{~g} / \mathrm{cm}^{3}\right)\end{array}$ \\
\hline 0.01 & $117.6 \pm 0.8$ & $21.04 \pm 0.03$ & $2.00 \pm 0.01$ \\
\hline 0.1 & $1.179 \pm 0.003$ & $0.227 \pm 0.0003$ & $0.162 \pm 0.001$ \\
\hline 1 & $0.0592 \pm 0.0004$ & $0.06240 \pm 0.00005$ & $0.06950 \pm 0.00009$ \\
\hline 10 & $0.03414 \pm 0.0004$ & $0.02240 \pm 0.00005$ & $0.0207 \pm 0.0001$ \\
\hline
\end{tabular}

The abilities of the scintillators to cause Compton scattering on gamma-ray as a function of incident photon energy is shown in figure 3.8. The thickness of the scintillators used in this set of simulations was fixed at $2.0 \mathrm{~cm}$. Compton scattering probability rises dramatically in the energy region $0.1 \mathrm{MeV}-1 \mathrm{MeV}$, before starting to fall again. The decrease in Compton scattering probability with increasing incident gamma-ray (> $1 \mathrm{MeV}$ ) is likely due to the decrease in the overall interaction probability. 


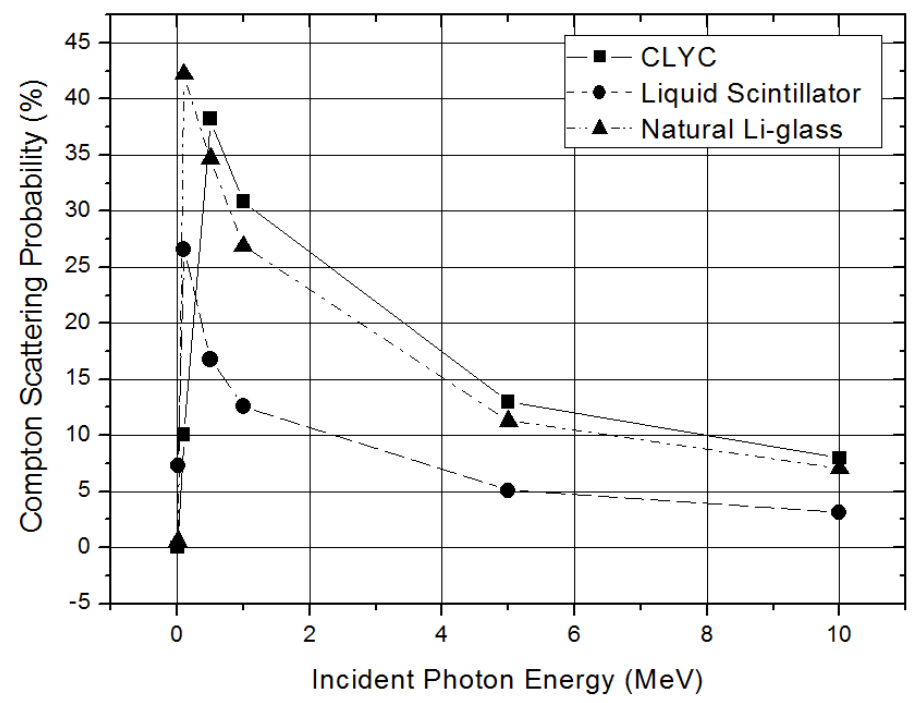

Figure 3.8 Comparison of Compton scattering probability as function of energy between CLYC, GS10 Natural Li-glass (GS10) and EJ-309 liquid scintillator.

\section{Designing a dual particle imaging system}

The results of the simulations in this work have been utilised in the designing process of a multilayered neutron/gamma imaging system. The simulations were used to find the scintillator material which exhibited optimum detection and attenuation capabilities for thermal neutrons, fast neutrons and gamma-rays. Moreover, the results were used to determine the optimum thickness of each layer in the design. Natural Li-glass (GS10) was found to be the optimum detector material for the design. The dimensions of the Li-glass (GS10) are the optimal design parameters derived from the data presented in figures 3.1 and 3.4. From the results, $10 \mathrm{~mm}$ thickness of Li-glass can capture up to $80 \%$ thermal neutrons while transmitting $92 \%$ of high energy gamma-rays. The thickness of the second layer was selected to be $15 \mathrm{~mm}$. This layer will serve as a second interaction plane of fast neutrons and a scattering plane of transmitted gamma-rays. A monolithic layer of a scintillator material, such as caesium iodide is added to the system to detect scattered gamma-rays. SiPM arrays will be used to detect resultant photons. The arrangement of the detectors and an early estimated configuration of the system is shown in figure 4.1 .

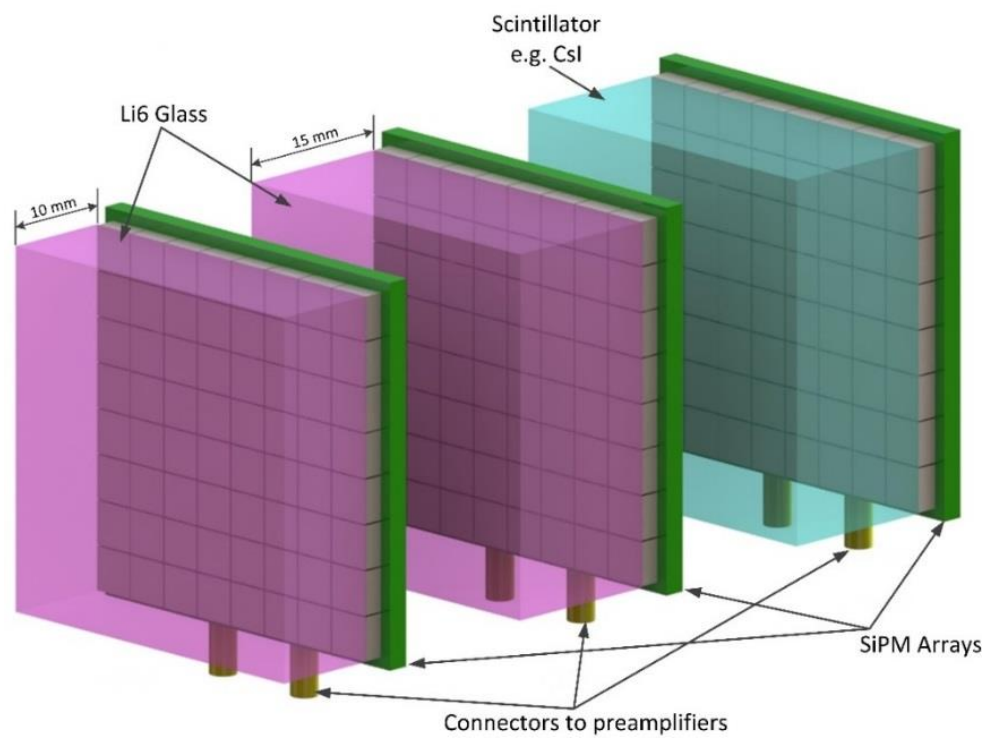

Figure 4.1 Configuration and dimensions of detectors in the novel design of a neutron/gamma imaging system. MCNP6.1.0 and Geant4 simulations showed that for this particular configuration, the system can detect up to $80 \%$ of thermal neutrons and up to $50 \%$ of gamma photons. Further investigations and experimental work are in progress. 


\section{Conclusion}

In this work CLYC, CLYC-6, natural Li-glass and EJ-309 scintillators were characterized by studying thermal neutron capture efficiency, fast neutron single elastic scattering abilities and gamma-ray attenuating abilities using two simulation toolkits, MCNP6.1.0 and Geant4.10.2. CLYC-6, and to a slightly lesser extent CLYC, showed impressive capturing efficiency of thermal neutrons with a maximum of $100 \%$. For thermal neutron detection efficiency, however, Li-glass scintillator exhibited the highest efficiency compared to the other three scintillators due to its higher atomic density of ${ }^{6} \mathrm{Li}$. The maximum capture efficiency of natural Li-glass was found to be around 99.95\%. EJ-309 liquid scintillator showed the lowest attenuation abilities at thicknesses lower than $5 \mathrm{~cm}$ with around $21.6 \%$. The Li-glass scintillator had the highest attenuation abilities of neutrons at $1 \mathrm{keV}$ although EJ-309 showed higher attenuation abilities at higher energies $(>10 \mathrm{keV})$. The probability of single scattering events for natural Li-glass is the highest of the three scintillators. CLYC and CLYC-6 showed the highest attenuation abilities of gamma-ray at energies lower than $1 \mathrm{MeV}$ mainly due to its high effective atomic number. At higher energies, the attenuation abilities of the four materials slightly varies when compared to one another. Natural Liglass scintillators and both elpasolite scintillators demonstrate good Compton scattering efficiency of gamma-rays with natural Li-glass acquiring slightly higher scattering efficiencies at energies lower than $1 \mathrm{MeV}$. The results achieved were used in designing a basic neutron/gamma imaging system which will be built and tested in work to follow on.

\section{Acknowledgments}

This work was supported by Lancaster University and sponsored by the Omani Government.

\section{References}

1. Katagiri, M., et al., Scintillation materials for neutron imaging detectors. Nuclear Instruments \&amp; Methods in Physics Research Section a-Accelerators Spectrometers Detectors and Associated Equipment, 2004. 529(1-3): p. 274-279.

2. Weber, M.J., Inorganic scintillators: today and tomorrow. Journal of Luminescence, 2002. 100(1-4): p. 35-45.

3. van Eijk, C.W.E., Inorganic Scintillators for Thermal Neutron Detection. Ieee Transactions on Nuclear Science, 2012. 59(5): p. 2242-2247.

4. Giaz, A., et al., The CLYC-6 and CLYC-7 response to gamma-rays, fast and thermal neutrons. Nuclear Instruments \& Methods in Physics Research Section a-Accelerators Spectrometers Detectors and Associated Equipment, 2016. 810: p. 132-139.

5. Yang, P., et al., The Synthesis and Structures of Elpasolite Halide Scintillators, in Nuclear Radiation Detection Materials-2009, M. Fiederle, et al., Editors. 2010. p. 185-192.

6. Glodo, J., et al., Selected Properties of Cs2LiYCl6, Cs2LiLaCl6, and Cs2LiLaYBr6 Scintillators. Ieee Transactions on Nuclear Science, 2011. 58(1): p. 333-338.

7. Tanner, P.A., et al., Absorption and emission spectra of Ce3+in elpasolite lattices. Journal of the American Chemical Society, 2003. 125(43): p. 13225-13233.

8. Gundiah, G., et al., Structure and scintillation properties of Ce3+-activated Cs2NaLaCl6, Cs3LaCl6, Cs2NaLaBr6, Cs3LaBr6, Cs2NaLaI6 and Cs3LaI6. Journal of Luminescence, 2014. 149: p. 374-384.

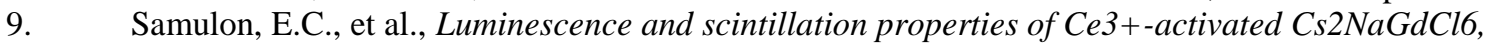
Cs3GdCl6, Cs2NaGdBr6 and Cs3GdBr6. Journal of Luminescence, 2014. 153: p. 64-72.

10. Kim, H.J., et al., Tl2LiYCl6 (Ce3+): New Tl-based Elpasolite Scintillation Material. Ieee Transactions on Nuclear Science, 2016. 63(2): p. 439-442.

11. Nattress, J., et al., Capture-gated Spectroscopic Measurements of Monoenergetic Neutrons with a Composite Scintillation Detector. Ieee Transactions on Nuclear Science, 2016. 63(2): p. 1227-1235.

12. Wang, C.L. and R.A. Riedel, Improved neutron-gamma discrimination for a Li-6-glass neutron detector using digital signal analysis methods. Review of Scientific Instruments, 2016. 87(1).

13. CHEN Yan-Ping, L.D.-L., Development of Containing $<$ sup $>6</$ sup $>$ Li Glass Scintillators for Neutron Detection. Journal of Inorganic Materials, 2012. 27(11): p. 1121-1128.

14. Lee, H.Y., et al., Li-glass detector response study with a Cf-252 source for low-energy prompt fission neutrons. Nuclear Instruments \& Methods in Physics Research Section a-Accelerators Spectrometers Detectors and Associated Equipment, 2013. 703: p. 213-219.

15. Arikawa, Y., et al., Multichannel down-scattered neutron detector for areal density measurement, in Ifsa 2011 - Seventh International Conference on Inertial Fusion Sciences and Applications, P. Mora, K.A. Tanaka, and E. Moses, Editors. 2013. 
16. Lampoudis, C., et al., Neutron transmission and capture cross section measurements for Am-241 at the GELINA facility. European Physical Journal Plus, 2013. 128(8).

17. Polack, J.K., et al., Dual-Particle Imager for Standoff Detection of Special Nuclear Material, in 2011 Ieee Nuclear Science Symposium and Medical Imaging Conference. 2011. p. 1494-1500.

18. Poitrasson-Riviere, A., et al., Dual-particle imaging system based on simultaneous detection of photon and neutron collision events. Nuclear Instruments \& Methods in Physics Research Section aAccelerators Spectrometers Detectors and Associated Equipment, 2014. 760: p. 40-45.

19. Klix, A., et al., Fast neutron and gamma-ray spectra measurements with a NE-213 spectrometer in the FNG Copper Benchmark Experiment. Fusion Engineering and Design, 2016. 109: p. 309-314.

20. Blanc, P., et al., Neutron/gamma pulse shape discrimination in plastic scintillators: Preparation and characterization of various compositions. Nuclear Instruments \& Methods in Physics Research Section a-Accelerators Spectrometers Detectors and Associated Equipment, 2014. 750: p. 1-11.

21. Bertrand, G.H.V., et al., Pulse shape discrimination between (fast or thermal) neutrons and gamma rays with plastic scintillators: State of the art. Nuclear Instruments \& Methods in Physics Research Section a-Accelerators Spectrometers Detectors and Associated Equipment, 2015. 776: p. 114-128.

22. Pellegri, L., et al., Performances of a 1 " x1 " Cs2LiYCl6 scintillator detector. 2013 Ieee Nuclear Science Symposium and Medical Imaging Conference (Nss/Mic), 2013.

23. Giaz, A., et al., Fast neutron measurements with Li-7 and Li-6 enriched CLYC scintillators. Nuclear Instruments \& Methods in Physics Research Section a-Accelerators Spectrometers Detectors and Associated Equipment, 2016. 825: p. 51-61.

24. Whitney, C.M., et al., Gamma-neutron imaging system utilizing pulse shape discrimination with CLYC. Nuclear Instruments \& Methods in Physics Research Section a-Accelerators Spectrometers Detectors and Associated Equipment, 2015. 784: p. 346-351.

25. Glodo, J., R. Hawrami, and K.S. Shah, Development of Cs2LiYCl6 scintillator. Journal of Crystal Growth, 2013. 379: p. 73-78.

26. Ban, G., et al., First tests of Li-6 doped glass scintillators for ultracold neutron detection. Journal of Research of the National Institute of Standards and Technology, 2005. 110(3): p. 283-288.

27. Popov, V., P. Degtiarenko, and Ieee, Lithium Glass Scintillator Neutron Detector as an Improved Alternative to the Standard He-3 Proportional Counter, in 2010 Ieee Nuclear Science Symposium Conference Record. 2010. p. 1819-1822.

28. Rich, G.C., et al., Fabrication and characterization of a lithium-glass-based composite neutron detector. Nuclear Instruments \& Methods in Physics Research Section a-Accelerators Spectrometers Detectors and Associated Equipment, 2015. 794: p. 15-24.

29. Yamazaki, A., et al., Neutron-gamma discrimination based on pulse shape discrimination in a Ce:LiCaAlF6 scintillator. Nuclear Instruments \& Methods in Physics Research Section a-Accelerators Spectrometers Detectors and Associated Equipment, 2011. 652(1): p. 435-438.

30. Tomanin, A., et al., Characterization of a cubic EJ-309 liquid scintillator detector. Nuclear Instruments \& Methods in Physics Research Section a-Accelerators Spectrometers Detectors and Associated Equipment, 2014. 756: p. 45-54.

31. Technology, E. 18/02/2016]; Available from: EJ-309 URL http://www.eljentechnology.com/index.php/products/liquid-scintillators/73-ej-309〉.

32. Enqvist, A., et al., Neutron light output response and resolution functions in EJ-309 liquid scintillation detectors. Nuclear Instruments \& Methods in Physics Research Section a-Accelerators Spectrometers Detectors and Associated Equipment, 2013. 715: p. 79-86.

33. Pino, F., et al., The light output and the detection efficiency of the liquid scintillator EJ-309. Applied Radiation and Isotopes, 2014. 89: p. 79-84.

34. van Eijk, C.W.E., Inorganic-scintillator development. Nuclear Instruments \& Methods in Physics Research Section a-Accelerators Spectrometers Detectors and Associated Equipment, 2001. 460(1): p. $1-14$.

35. Poitrasson-Riviere, A., et al., Angular-resolution and material-characterization measurements for a dual-particle imaging system with mixed-oxide fuel. Nuclear Instruments \& Methods in Physics Research Section a-Accelerators Spectrometers Detectors and Associated Equipment, 2015. 797: p. 278-284.

36. Ayaz-Maierhafer, B., et al., Angular resolution study of a combined gamma-neutron coded aperture imager for standoff detection. Nuclear Instruments \& Methods in Physics Research Section aAccelerators Spectrometers Detectors and Associated Equipment, 2013. 712: p. 120-125.

37. Goorley, J.T.J., Michael R.; Booth, Thomas E.; Brown, Forrest, et al., Initial MCNP6 Release Overview - MCNP6 version 1.0. 2012. 
38. Agostinelli, S., et al., GEANT4-a simulation toolkit. Nuclear Instruments \& Methods in Physics Research Section a-Accelerators Spectrometers Detectors and Associated Equipment, 2003. 506(3): p. 250-303.

39. Allison, J., et al., Geant4 developments and applications. Ieee Transactions on Nuclear Science, 2006. 53(1): p. 270-278.

40. Allison, J., et al., Recent developments in GEANT4. Nuclear Instruments \& Methods in Physics Research Section a-Accelerators Spectrometers Detectors and Associated Equipment, 2016. 835: p. 186-225.

41. Reuss, P., Neutron physics. 2008, France: EDP Science.

42. NEA, O. Java-based nuclear data information system. 16/01/2016 [cited 2016 10/02/2016]; Available from: http://www.oecd-nea.org/janis/.

43. Kahn, F., The physics of radiation therpy. 3rd ed. 2003: Lippincott Williams \& Wilkins, Philadelphia USA.

44. Taylor, M.L., et al., The effective atomic number of dosimetric gels. Australasian Physical \& Engineering Sciences in Medicine, 2008. 31(2): p. 131-138.

45. Knoll, G.F., Radiation detection and measurment. Fourth ed. 2010, United States of America: John Wiley and Sons.

46. Colonna, N. and S. Altieri, Simulations of neutron transport at low energy: A comparison between GEANT and MCNP. Health Physics, 2002. 82(6): p. 840-846.

47. Enger, S.A., et al., Monte Carlo calculations of thermal neutron capture in gadolinium: A comparison of GEANT4 and MCNP with measurements. Medical Physics, 2006. 33(2): p. 337-341.

48. Yeh, Y.S., et al., Simulating neutron propagations with FLUKA, GEANT4 and MCNP, in 2007 Ieee Nuclear Science Symposium Conference Record, Vols 1-11. 2007. p. 2016-+.

49. Guardiola, C., et al., Geant4 and MCNPX simulations of thermal neutron detection with planar silicon detectors. Journal of Instrumentation, 2011. 6.

50. Monk, S.D., et al., A comparison of MCNP6-1.0 and GEANT 4-10.1 when evaluating the neutron output of a complex real world nuclear environment: The thermal neutron facility at the Tri Universities Meson facility. Nuclear Instruments and Methods in Physics Research, Section B: Beam Interactions with Materials and Atoms, 2017. 399: p. 48-61.

51. Berger, J., Hubbell, J.H., Seltzer, S.M., Chang, J., Coursey, J.S. Sukumar, R., Zucker, D.S. and Olsen, K. XCOM: Photon Cross Sections Database. 2009 19/03/2015 [cited 2016 08/02/2016]; Available from: http://www.nist.gov/pml/data/xcom/index.cfm. 